\title{
Chemical Investigation of Wrightia tinctoria
}

\section{Sajitha $\mathbf{M}^{1}$ and Subramani $\mathbf{K}^{2^{*}}$}

${ }^{1}$ Department of Chemistry, Kanchimamunivar Centre for Post Graduate Studies, Puducherry, India

${ }^{2}$ Department of Chemistry, Barathidasan Government College for Women, Puducherry, India

"Corresponding author: Subramani K, Department of Chemistry, Barathidasan Government College for Women, Puducherry, India, Tel: 9894437687; E-mail: subbu0302@gmail.com

Received: September 22, 2017; Accepted: October 06, 2017; Published: October 16, 2017

Copyright: (C) 2017 Sajitha M, et al. This is an open-access article distributed under the terms of the Creative Commons Attribution License, which permits unrestricted use, distribution, and reproduction in any medium, provided the original author and source are credited.

\begin{abstract}
The systematic chemical analysis of leaves of Wrightia tinctoria were found to contain two flavonoid glycosides Kaempferol 3-O-rhamnoside and Quercetin 3-O-sophoroside and two flavonoid aglycone Kaempferol and Quercetin. The detailed UV ${ }^{1} \mathrm{H}^{13} \mathrm{C}$ NMR and Mass spectral data confirm the characterization of the above compounds. All these compounds are reported first time from the leaves of Wrightia tinctoria.
\end{abstract}

Keywords: Wrightia tinctoria; Flavonoid; Quercetin 3-0-sophoroside

\section{Introduction}

Wrightia tinctoria R. Br belongs to family Apocynaceae commonly called as Sweet Indrajao, Pala Indigo Plant, Dyer's Oleander "Jaundice Curvative tree in South India. It is distributed throughout the world and occurs abundantly in India and Burma. In deciduous forests, especially in Rajasthan, Madhya Pradesh and Peninsular India [1]. The bark of the Wrightia tinctoria is considered for antidiarrhoeal, aphrodisiac, anthelmintic, febrifuge, stomachic, toothache, tonic and dog bite [2-4]. It has got very important place in traditional healing and also is widely recognized medicinal plant [5]. Oil 777 prepared out of the fresh leaves of the plant has been assigned to analgesic, antiinflammatory and antipyretic activities and to be effective in the treatment of psoriasis [6-8]. It has anti-dandruff properties and hence used in hair oil preparations [9].

The vast number of literature found in database revealed that the extracts of different parts of Wrightia tinctoria showed significant pharmacological actions. Because there is a need for further investigations to isolate active principles that confer pharmacological action in continuation of our studies in the flavonoids of Indian medicinal plants, the leaves of Wrightia tinctoria were investigated for flavonoids and the results leading to the isolation of Kaempferol-3-Orhamnoside, Quercetin 3-O-sophoroside, Kaempferol and Quercetin.

\section{Results and Discussion}

From the alcoholic extract of the air-dried leaves, four flavonoids were isolated and characterized.

\section{Compound (1)}

Compound (1) was purple under UV changing to yellow under $\mathrm{UV} / \mathrm{NH}_{3}$ and had $\lambda$ max and $\mathrm{R}_{\mathrm{f}}$ values characterestics of a flavanol glycoside. The presence of free 5,7 and 4' $\mathrm{OH}$ groups can be established by the UV spectrum of the compound with shift reagents. The ${ }^{1} \mathrm{H}$ NMR spectrum of the compound showed $\delta 6.44\left[{ }^{1} \mathrm{H}, \mathrm{dJ}=1.76 \mathrm{~Hz}\right]$ and $\delta 6.2\left[{ }^{1} \mathrm{H}, \mathrm{dJ}=1.72 \mathrm{~Hz}\right]$ which was predicted by the hydrogens at $\mathrm{C}-8$ and C- 6 of the A ring of the flavonoid skelton and two signals with ortho coupling at $\delta 6.9[2 \mathrm{H}, \mathrm{dJ}=8.74 \mathrm{~Hz}]$ and $\delta 7.9[2 \mathrm{H}, \mathrm{dJ}=8.76 \mathrm{~Hz}]$ for the hydrogens at C-3', C-5', C-2' and C-6'of the B-ring. Presence of a sugar moiety can be characterized by the presence of an anomeric hydrogen signal at $\delta 5.3\left[{ }^{1} \mathrm{H}, \mathrm{dJ}=2 \mathrm{~Hz}\right]$ and the appearance of an anomeric carbon signal at $\delta 103.5$ in the ${ }^{13} \mathrm{C}$ NMR spectrum. The methyl signal observed at $\delta 1.1[3 \mathrm{H}, \mathrm{S}]$ in the $1 \mathrm{H}$ NMR spectrum and at 18.3 in the ${ }^{13} \mathrm{C}$ NMR spectrum indicated that the sugar moiety was rhamnose. On EIMS, it gave a peak at $\mathrm{m} / \mathrm{z} 430$ which was in good agreement with the molecular formula $\mathrm{C}_{21} \mathrm{H}_{19} \mathrm{O}_{10}$. From these data, compound (1) was characterized as Kaempferol-3-Orhamnopyranoside.<smiles>[R20]c1cc(O)c2c(=O)c(O)c(-c3ccc(O)c([R9])c3)oc2c1</smiles>

Compound (1) $\mathrm{R}_{1}=$ rhamnoside, $\mathrm{R}_{2}, \mathrm{R}_{3}=\mathrm{H}$

Compound (2) $\mathrm{R}_{1}=$ sophoroside, $\mathrm{R}_{2}=\mathrm{H}$ and $\mathrm{R}_{3}=\mathrm{OH}$

Compound (3) $\mathrm{R}_{1}, \mathrm{R}_{2}$ and $\mathrm{R}_{3}=\mathrm{H}$

Compound (4) $\mathrm{R}_{1}$ and $\mathrm{R}_{2}=\mathrm{H}, \mathrm{R}_{3}=\mathrm{OH}$

\section{Compound (2)}

It gave yellow colour with alkalis. Pink with $\mathrm{Mg}-\mathrm{HCl}$ Greenish brown with $\mathrm{Fe}^{3+}$ Positive Molisch's test indicating it to be a flavonoid glycoside. It was purple under UV changing to yellow in $\mathrm{UV} / \mathrm{NH}_{3}$ and 
had $\lambda \max (\mathrm{MeOH}) 257,286 \mathrm{sh}, 358.5 \mathrm{~nm}$ and $\mathrm{R}_{\mathrm{f}}$ typical of flavonol glycoside. Evidence of presence of ortho dihydroxyl group in $\mathrm{B}$ ring was obtained from a hyposochromic shift of $25 \mathrm{~nm}$ in Band I of $\mathrm{AlCl}_{3} / \mathrm{HCl}$ relative to $\mathrm{AlCl}_{3}$ spectrum. ${ }^{1} \mathrm{H}$ NMR spectrum showed the evidence of 3,5,7,3', '-penta oxygenated flavone as well as the presence of protons at 2,6,5,6,8 by a typical doublet pattern. The characteristic signals in the aliphatic region were assigned to the anomeric proton and other sugar protons showing the compound 2 has a diglycoside of 3,5,7,3'4'-pentaoxygenated flavone. The compound gave a molecular ion peak at $\mathrm{m} / \mathrm{z}=626 \mathrm{~g} / \mathrm{mol}$ in the EIMS. On EIMS, it gave a peak at $\mathrm{m} /$ $\mathrm{z}=301$ which was in good agreement with the molecular formula $\mathrm{C}_{15} \mathrm{H}_{9} \mathrm{O}_{7}$ of aglycone. These discussions led to the identification of the compound (2) was Quercetin 3-O-sophoroside.

\section{Compound (3)}

Compound (3) $\mathrm{C}_{15} \mathrm{H}_{10} \mathrm{O}_{6}, \mathrm{mp} 277-279^{\circ} \mathrm{C}$ gave pink colour with $\mathrm{Mg}$ $\mathrm{HCl}$, yellow with alkalis and green with $\mathrm{Fe}^{3+}$. It was yellow under UV and $\mathrm{UV} / \mathrm{NH}_{3}$ characteristic of flavonol with free 3-OH. The presence of free 5,7 and 4' OH groups can be established by the UV spectrum of the compound with shift reagents. A hypsochromic shift of only $8 \mathrm{~nm}$ in band $\mathrm{I}$ of $\mathrm{AlCl}_{3} / \mathrm{HCl}$ spectrum compared to $\mathrm{AlCl}_{3}$ spectrum revealed the absence of orthodihydroxyl in ring B. Further the ${ }^{1} \mathrm{H}$ NMR spectrum showed signals at $\delta 12.6$ for $5-\mathrm{OH}, 10.1$ for $7-\mathrm{OH}, 7.6$ (d) for H- 2,6', 6.92 (d) for H- 3,'5, 6.44 (d) for H-8 and 6.19 (d) for H-6 confirms the above facts. It was also supported by ${ }^{13} \mathrm{CNMR}$ spectrum of the compound (3). From these data compound (3) was characterized as 3,5,7,4'-tetrahydroxy flavone (Kaempferol) whose identity was further confirmed by direct comparison including co-PC with an authentic sample [10].

\section{Compound (4)}

Compound (4) had UV fluorescence and UV maxima characteristic of an aglycone flavanol. ${ }^{1} \mathrm{H}$ NMR spectrum showed the evidence of $3,5,7,3^{\prime}, 4^{\prime}$ - penta oxygenated flavone as well as the presence of protons at $2,6,5,6,8$ by a typical doublet pattern. The ${ }^{13} \mathrm{C}$ NMR spectrum of the compound further supported the above findings. Thus the structure of the flavonoid was established as a 3,5,7,3',4'-penta hydroxy flavone or Quercetin. It was further confirmed by the direct comparison with an authentic sample from Berberis aristata [11].

\section{Experimental}

\section{Plant material}

Fresh leaves $(1 \mathrm{Kg})$ were collected from Lawspet, Pondicherry on July 2014 and authenticated by the Department of Botany, K.M. Centre for P.G. Studies, Pondicherry were a voucher specimen was deposited.

\section{Extraction and isolation}

The air-dried leaves of the plant were extracted thrice with boiling $95 \% \mathrm{EtOH}(3 \mathrm{X} 5 \mathrm{~L})$ and concentrated in vacuo to $400 \mathrm{~mL}$. The aqueous extract was fractionated into Benzene, Ether, Ethyl acetate and Methyl Ethyl ketone solubles. The Benzene and ether fraction gave no characteristic spots for flavonoids and hence was not worked up further. The EAc and MEK concentrate on paper chromatography were found to contain same compounds. Hence these two were mixed and were chromatographed over a coloumn of Sephadex LH-20 using $\mathrm{MeOH}$ as solvent. 45 fractions of each $10 \mathrm{ml}$ were collected. Compound (1) $36 \mathrm{mg}$ from fractions (5-12), Compound (2) $388.9 \mathrm{mg}$ from fractions (17-29), Compound (3) $86 \mathrm{mg}$ from fractions (30-35) and Compound (4)126.6 mg from fractions (37-43) were obtained.

\section{Kaempferol-3-O-rhamnopyranoside (1)}

Reddish Brown coloured needles from $\mathrm{Me}_{2} \mathrm{CO}, \mathrm{mp} 152-153^{\circ} \mathrm{C}(36$ mg), $\mathrm{C}_{21} \mathrm{H}_{19} \mathrm{O}_{10}$, gave pink colour with $\mathrm{Mg}-\mathrm{HCl}$, olive green with $\mathrm{Fe}^{3+}$ and yellow with alkalis. It was purple under UV and changing yellow under $\mathrm{UV} / \mathrm{NH}_{3}$. UV ( $\lambda$ max, nm) $(\mathrm{MeOH}): 266,351 ;(+\mathrm{NaOAc}): 274$, 315.5 sh, 376.5; $\left(+\mathrm{NaOAc} / \mathrm{H}_{3} \mathrm{BO}_{3}\right): 263.5,366.5 ;\left(+\mathrm{AlCl}_{3}\right): 273.5$, 346.5, 407.5; $\left(+\mathrm{AlCl}_{3} / \mathrm{HCl}\right): 267,346.5,392 ;(+\mathrm{NaOMe}): 274,317 \mathrm{sh}$, $391.5 ;{ }^{1} \mathrm{H}$ NMR (300 MHz, DMSO-d 6 , $\left.\delta \mathrm{ppm}\right): 12.6$ (s, $\left.{ }^{1} \mathrm{H}, \mathrm{OH}-7\right)$, $11.2\left({ }^{1} \mathrm{H}, \mathrm{OH}-5\right), 7.9\left(\mathrm{~d}, \mathrm{~J}=8.76 \mathrm{~Hz},{ }^{2} \mathrm{H}, \mathrm{H}-2^{\prime}, 6^{\prime}\right), 6.9\left(\mathrm{~d}, \mathrm{~J}=8.74 \mathrm{~Hz},{ }^{2} \mathrm{H}\right.$, H-3,5') 6.44 (d, J=1.76 Hz, $\left.{ }^{1} \mathrm{H}, \mathrm{H}-8\right), 6.2$ (d, J=1.72 Hz, $\left.{ }^{1} \mathrm{H}, \mathrm{H}-6\right)$, of aglycone: 5.3 (d, J=2 Hz, ${ }^{1} \mathrm{H}, \mathrm{H}-1$ "), 4.42 (dd, J=3.4, $1.5 \mathrm{~Hz},{ }^{1} \mathrm{H}, \mathrm{H}-2$ "), $3.56\left(\mathrm{~m},{ }^{1} \mathrm{H}, \mathrm{H}-3\right.$ ", 4",5"), $1.06\left(\mathrm{~d}, \mathrm{~J}=6.3 \mathrm{~Hz},{ }^{3} \mathrm{H}, \mathrm{H}-6\right.$ ") of rhamnose. ${ }^{13} \mathrm{C}$ NMR (75.48 MHz, DMSO-d $\left.{ }_{6}-\mathrm{CDCl}_{3}, \delta, \mathrm{ppm}\right) 177.9$ (C-4), 164.3 (C-7), 161.6 (C-5), 156.9 (C-9), 145.3 (C-4'), (C-2), 133.8 (C-3), 131.4 (C-1'), 131.3(C-3', 5'), 115.7 (C-2', 6'), 115.5 (C-2'), 104.4 (C-10), 101.9 (C-1'), 99.2 (C-6), 94.045 (C-8), 72.4 (C-4"), 71.6 (C-2"), 70.9 (C-3"), 68.7(C-5"), 18.3 (C-6"); MS (electrospray, relative intensity as\%) 430 $\left(\mathrm{M}-\mathrm{H}^{+}, 18\right)$.

\section{Quercetin 3-O-sophoroside (2)}

Yellow needles from $\mathrm{Me}_{2} \mathrm{CO}$, (388.9 mg), $\mathrm{C}_{27} \mathrm{H}_{30} \mathrm{O}_{17}$, gave tomato red colour with $\mathrm{Mg}-\mathrm{HCl}$, olive green with $\mathrm{Fe}^{3+}$ and yellow with alkalis. It was purple under $\mathrm{UV}$ changing yellow under $\mathrm{UV} / \mathrm{NH}_{3}$. UV $(\lambda \max$, $\mathrm{nm})(\mathrm{MeOH}): 257,286 \mathrm{sh}, 358.5 ;(+\mathrm{NaOAc}): 267.5,378 ;(+\mathrm{NaOAc} /$ $\left.\mathrm{H}_{3} \mathrm{BO}_{3}\right): 261,376.5 ;\left(+\mathrm{AlCl}_{3}\right): 277,433 ;\left(+\mathrm{AlCl}_{3} / \mathrm{HCl}\right): 277,341,416$; $(+\mathrm{NaOMe}): 275,323,356 ;{ }^{1} \mathrm{H}$ NMR $\left(300 \mathrm{MHz}, \mathrm{DMSO}-\mathrm{d}_{6}, \delta \mathrm{ppm}\right)$ $12.64\left(\mathrm{~s},{ }^{1} \mathrm{H}, \mathrm{OH}-5\right) 10.99\left(\mathrm{~s},{ }^{1} \mathrm{H}, \mathrm{OH}-7\right) 9.84\left(\mathrm{~s},{ }^{1} \mathrm{H}, \mathrm{OH}-4{ }^{\prime}\right) 9.28\left(\mathrm{~s},{ }^{1} \mathrm{H}\right.$, OH-3) 8.06 (dd, J=2.2 Hz, ${ }^{1} \mathrm{H}, \mathrm{H}-2$ ') 7.6 (dd, J=8.3 Hz, ${ }^{1} \mathrm{H}, \mathrm{H}-6$ ') 6.85 (dd, J=8.4 Hz, ${ }^{1} \mathrm{H}, \mathrm{H}-5$ ) $6.41\left(\mathrm{~d}, \mathrm{~J}=2.2 \mathrm{~Hz},{ }^{1} \mathrm{H}, \mathrm{H}-8\right) 6.21(\mathrm{~d}, \mathrm{~J}=2.2 \mathrm{~Hz}$, $\left.{ }^{1} \mathrm{H}, \mathrm{H}-6\right)$, of aglycone ; 5.47 (d, J=7.6 Hz, ${ }^{1} \mathrm{H}, \mathrm{H}-1$ ”), $5.3(\mathrm{~d}, \mathrm{~J}=8.8 \mathrm{~Hz}$, ${ }^{1} \mathrm{H}, \mathrm{H}-2$ "), 5.0 (m, 4H, H-3", H-4", H-6"), 3.24 (m, $\left.1 \mathrm{H}^{1} \mathrm{H}, \mathrm{H}-5^{\prime \prime}\right)$, ofglucose; 5.39 (d,J=2Hz, ${ }^{1} \mathrm{H}, \mathrm{H}-1$ "'), $4.4\left(\mathrm{~d}, \mathrm{~J}=8.8 \mathrm{~Hz},{ }^{1} \mathrm{H}, \mathrm{H}-2{ }^{\prime \prime}\right), 3.45$ (m,2H,H-3"',H-4"',H-6"'), 3.09 (m, $\left.{ }^{1} \mathrm{H}, \mathrm{H}-5^{\prime \prime \prime}\right)$ of glucose. ${ }^{13} \mathrm{C}$ NMR (75.48 MHz, DMSO-d ${ }_{6}-\mathrm{CDCl}_{3}, \delta$,ppm) 177.9 (C-4), 164.6 (C-7), 161.7 (C-5), 156.8 (C-9), 156.7 (C-2), 148.9 (C-4'), 145.3 (C-3'), 133.8 (C-3), 122.4 (C-1'), 121.6 (C-6'), 116.7 (C-5'), 116.4 (C-2'), 104.4(C-10), 102.3 (C-1"), 101.3(C-1") 99.1(C-6), 93.9 (C-8), 78.0 (C-2"), 76.9 (C-3"), 76.3 (C-3"'), 74.6 (C-5”), 73.6 (C-5"'), 71.6 (C-2"”), 70.3 (C-4”), 68.3 (C-4"'), 61.4 (C-6"), 60.6 (C-6"'). MS (electrospray, relative intensity as \%) $625\left(\mathrm{M}-\mathrm{H}^{+}, 10\right), 301\left[\mathrm{M}^{+}\right.$- diglucose, 9$]$.

\section{Kaempferol (3)}

Crystallized as yellow needles from $\mathrm{Me}_{2} \mathrm{CO}, \mathrm{mp} .277-279^{\circ} \mathrm{C} \mathrm{C}_{15}$ $\mathrm{H}_{10} \mathrm{O}_{6}(86 \mathrm{mg})$. It gave pink colour with $\mathrm{Mg}$ - $\mathrm{HCl}$, yellow with alkalis and green with $\mathrm{Fe}^{3+}$. It was yellow under $\mathrm{UV}$ and $\mathrm{UV} / \mathrm{NH}_{3} \cdot \mathrm{UV}(\lambda$ max., $\mathrm{nm}) \mathrm{MeOH}: 266,360$; NaOAc : 271.6, 315.5 sh, 371.2 ; $\mathrm{NaOAc} / \mathrm{H}_{3} \mathrm{BO}_{3}: 262,362 ; \mathrm{AlCl}_{3}: 273.5,345,408.8 ; \mathrm{AlCl}_{3} / \mathrm{HCl}: 261.7$, 343,400 ; NaOMe : 271.8 , $325 \mathrm{sh}, 392.8{ }^{1} \mathrm{H}$ NMR (200 MHz, DMSO$\left.\mathrm{d}_{6}, \delta, \mathrm{ppm}\right) 12.5$ (s, $\left.{ }^{1} \mathrm{H}, \mathrm{OH}-5\right), 10.1$ (brs, $\left.{ }^{1} \mathrm{H}, \mathrm{OH}-7\right), 8.04(\mathrm{~d}, \mathrm{~J}=8.76 \mathrm{~Hz}$, $\left.2 \mathrm{H}, \mathrm{H}-2^{\prime}, 6^{\prime}\right), 6.92\left(\mathrm{~d}, \mathrm{~J}=8.76 \mathrm{~Hz}, 2 \mathrm{H}, \mathrm{H}-3^{\prime}, 5^{\prime}\right), 6.44$ (d, J=1.72 Hz, 1H, $\mathrm{H}-8$ ), 6.19 (d, J=1.74 Hz, $\left.{ }^{1} \mathrm{H}, \mathrm{H}-6\right) .{ }^{13} \mathrm{C}$ NMR (50 MHz, DMSO-d 6 , $\delta$, ppm) 176.00 (C-4), 164.0 (C-7), 160.81 (C-5), 159.28 (C-4'), 156.27 (C-9), 146.88 (C-2), 135.77 (C-3), 129.62 (C-2'6'), 121.78 (C-1'), 115.54 (C-3'5'), 103.14 (C-10), 98.31 (C-6), 93.59 (C-8). MS (electrospray, relative intensity as\%) $285\left(\mathrm{M}-\mathrm{H}^{+}, 89\right)$. 


\section{Quercetin (4)}

Yellow needles from $\mathrm{Me}_{2} \mathrm{CO}, \mathrm{mp} 305-306^{\circ} \mathrm{C}(126.6 \mathrm{mg}), \mathrm{C}_{15} \mathrm{H}_{10} \mathrm{O}_{7}$, gave pink colour with $\mathrm{Mg}-\mathrm{HCl}$, olive green with $\mathrm{Fe}^{3+}$ and yellow with alkalis. It was yellow under $\mathrm{UV}$ as well as $\mathrm{UV} / \mathrm{NH}_{3}$. UV $(\lambda$ max, nm) $(\mathrm{MeOH}): 255,305$ sh, 370; (+NaOAc): 274, 318, 386 (dec); (+NaOAcl $\left.\mathrm{H}_{3} \mathrm{BO}_{3}\right): 259,324 \mathrm{sh}, 380 ;(+\mathrm{AlCl} 3): 270,347,437 ;\left(+\mathrm{AlCl}_{3} / \mathrm{HCl}\right): 264$, 303 sh, 356, 424; (+NaOMe): 274, 326, 400 (dec); ${ }^{1} \mathrm{H}$ NMR $(300 \mathrm{MHz}$, DMSO-d ${ }_{6}, \delta$ ppm) $12.5\left(\mathrm{~s},{ }^{1} \mathrm{H}, \mathrm{OH}-5\right) 10.79\left(\mathrm{~s},{ }^{1} \mathrm{H}, \mathrm{OH}-7\right) 10.1\left(\mathrm{~s},{ }^{1} \mathrm{H}\right.$, OH-4') 9.6 (s, $\left.{ }^{1} \mathrm{H}, \mathrm{OH}-3\right) 9.36$ (s, $\left.{ }^{1} \mathrm{H}, \mathrm{OH}-3^{\prime}\right) 8.06\left(\mathrm{~d}, \mathrm{~J}=2.2 \mathrm{~Hz},{ }^{1} \mathrm{H}\right.$, H-2') 7.68 (d, J=8.3 Hz, ${ }^{1} \mathrm{H}, \mathrm{H}-6$ ') 7.55 (d, J=8.4 Hz, $\left.{ }^{1} \mathrm{H}, \mathrm{H}-5^{\prime}\right) 6.92(\mathrm{~d}$, $\left.\mathrm{J}=2.2 \mathrm{~Hz},{ }^{1} \mathrm{H}, \mathrm{H}-8\right) 6.42\left(\mathrm{~d}, \mathrm{~J}=2.2 \mathrm{~Hz},{ }^{1} \mathrm{H}, \mathrm{H}-6\right):{ }^{13} \mathrm{C} \mathrm{NMR}(75.48 \mathrm{MHz}$, DMSO-d ${ }_{6}-\mathrm{CDCl}_{3}, \delta$,ppm) $176.4(\mathrm{C}-4), 164.3$ (C-7), 161.2 (C-5), 156.5 (C-9), 148.2 (C-4'), 147.3 (C-2), 145.5 (C-3'), 136.1(C-3), 122.4 (C-1'), 120.4 (C-6'), 115.9 (C-5'), 115.5 (C-2'), 103.5 (C-10), 98.7 (C-6), 93.8 $\mathrm{s}(\mathrm{C}-8)$. MS (electrospray, relative intensity as\%) $301\left(\mathrm{M}-\mathrm{H}^{+}, 100\right), 285$ $\left[\mathrm{M}-\mathrm{OH}^{-}, 60\right]$,

\section{Acknowledgement}

The Authors are very thankful to the department of Botany, K.M. Centre for P.G. Studies, Puducherry for having authenticated the fresh leaves are collected from Lawspet Puducherry in July 2014.

\section{References}

1. Sivarajan VV, Balachandran I (1999) Ayurvedic drugs and their plant sources. Oxford and IBH Publishing Co.Pvt. Ltd. New Delhi, pp: 267-269.
2. Chopra RN, Nayar SI, Chopra IC (1956) Glossary of Indian medicinal plants. CSIR, p: 259.

3. Agarwal CK (1986) Economic Plants of India. Kailash Prakashan, Culcutta, p: 406.

4. Joshi SG (2000) Medicinal Plants. Oxford and IBH Publishing Co. Pvt. Ltd., New Delhi, p: 51.

5. Khyade MS, Vaikos NP (2011) Comparative phytochemical and antibacterial studies on the bark of Wrightia tinctoria and Wrightia arborea. Internat J Pharma Biosci 2: 176-181.

6. Kothari MJ, Rao KM (2000) Ethnobotanical studies of Thane District Maharashtra, Ethanobotany and Medicinal plants of Indian subcontinent. Scientific Publisher, Jodhpur, India.

7. Ghosh D, Thejmoorth P, Veluchamy G (1985) Anti-inflammatory, analgesic and antipyretic activities of 777 oil- a siddha medicine. Bull Med Ethnobot Res 6: 141-154.

8. Krishnamurthi JR, Kalaimani S, Veluchamy G (1981) Clinical study of vetapalai (Wrightia tinctoria) oil in the treatment of Kalanjagapadai (Psoriasis). Journ Res Ayur Siddha 2: 58-66.

9. http://www.worldagroforestry.org/

10. Subramanian SS, Nair AGR (1970) Chlorogenin and kaempferol glycosides from the flowers of Agave americana. Phytochem 9: 2582.

11. Nair AGR, Siva Kumar K (1992) Non-identity of hortensin from Millingtonia hortensis with 3, 4'-dihydroxy-6, 7-dimethoxyflavone. Fitotera 63: 189 . 